\title{
COVARIANT COMPUTATION IN \\ SPECIAL RELATIVISTIC DYNAMICS
}

\author{
by \\ Donald Greenspan
}

TR \#298

\section{DATE DUE}

Mathema $s$ Department

University o Texas at Arlington 
COVARIANT COMPUTATION IN SPECIAL RELATIVISTIC DYNAMICS

by

Donald Greenspan

TR \#298

\section{ABSTRACT}

The motion of a particle in a special relativistic framework is studied numerically. Difference equations which are covariant are developed first. It is then shown how computations can be done in both the lab and rocket frames so that the numerical results are related by the Lorentz transformation. Finally, assuming the usual force formula for harmonic oscillation, it is shown that the dynamical equation is necessarily nonlinear and trajectories are studied numerically for various initial data. 
1. Introduction Most popular numerical methods, like Runge-Kutta, multistep, and Taylor expansion methods, when applied to approximate solutions of second order, nonlinear, Newtonian initial value problems, do not preserve fundamental physical invariants, like energy and momentum, when these are present. However, specialized implicit methods are now available which do preserve such invariants [1]. If one considers second order, nonlinear, special relativistic initial value problems, it would be important to have a numerical method which, when applied on identical computers in the $\mathrm{lab}$ and rocket frames, would yield numerical results which are connected by the Lorentz transformation. In this paper, we show how this can be done in a way that is consistent with recent models of discrete space-time $[2-7]$.

2. Relativistic Considerations Consider two one-dimensional, inertial frames, coincident at time $t=0$, denoted by $X$ and $X^{\prime}$. Let $X^{\prime}$ be in motion in the $x$ direction with speed $u$ relative to the $X$ frame. The $X$ frame is called the lab frame and the $X^{\prime}$ frame the rocket frame. All the usual assumptions about $\mathrm{lab}$ and rocket frames [8 - 12] are appropriated here. In particular, we have the Axiom of Relativity, that is, that all laws of physics and all physical constants, including the speed of light $c$, are the same in every initial reference frame. The Lorentz transformation connecting events $(x, t)$ in the lab frame and $\left(x^{\prime}, t^{\prime}\right)$ in the rocket frame are

$$
x^{\prime}=\frac{c(x-u t)}{\left(c^{2}-u^{2}\right)^{\frac{1}{2}}} \quad t^{\prime}=\frac{c^{2} t-u x}{c\left(c^{2}-u^{2}\right)^{\frac{1}{2}}}
$$

or, equivalently,

$$
x=\frac{c\left(x^{\prime}+u t^{\prime}\right)}{\left(c^{2}-u^{2}\right)^{\frac{1}{2}}}, t=\frac{c^{2} t^{\prime}+u x^{\prime}}{c\left(c^{2}-u^{2}\right)^{\frac{1}{2}}}
$$

Next, recall that all velocities to be considered will satisfy $|v|<c$. Finally, recall that the motion $P$ of a particle of mass $m$, acted upon by a force $F$, is given in the lab frame by

$$
F=\frac{d}{d t}(m v), \quad m=\frac{m_{o}}{\sqrt{1-\left(\frac{v}{c}\right)^{2}}}
$$

and, covariantly, in the rocket frame by 


$$
F^{\prime}=\frac{d}{d t^{\prime}}\left(m^{\prime} v^{\prime}\right), \quad m^{\prime}=\frac{m_{o}}{\sqrt{1-\left(\frac{v^{\prime}}{c}\right)^{2}}} .
$$

3. Numerical Methodology In addition to identical clocks, we assume now that the observers in the lab and rocket frames have identical computers. In the lab, let $\Delta t>0$ and $t_{k}=k \Delta t, k=0,1, \cdots$. At time $t_{k}$, let $P$ be at $x_{k}$ in the lab. Then, in the rocket, $P$ will be at $x_{k}^{\prime}$ at time $t_{k}{ }^{\prime}$, where

$$
x_{k}{ }^{\prime}=\frac{c\left(x_{k}-u t_{k}\right)}{\left(c^{2}-u^{2}\right)^{\frac{1}{2}}}, \quad t_{k}{ }^{\prime}=\frac{c^{2} t_{k}-u x_{k}}{c\left(c^{2}-u^{2}\right)^{\frac{1}{2}}}
$$

or equivently,

$$
x_{k}=\frac{c\left(x_{k}{ }^{\prime}+u t_{k}{ }^{\prime}\right)}{\left(c^{2}-u^{2}\right)^{\frac{1}{2}}}, t_{k}=\frac{c^{2} t_{k}{ }^{\prime}+u x_{k}{ }^{\prime}}{c\left(c^{2}-u^{2}\right)^{\frac{1}{2}}}
$$

The validity of (3.1) and (3.2) follows because they are merely special cases of (2.1a) and (2.1b), that is, they result from the particular choices $x=x_{k}, t=t_{k}$.

The concepts of velocity and acceleration are now introduced as follows. At $t_{k}$ in the lab, set

$$
v_{k}=\frac{\Delta x_{k}}{\Delta t_{k}}=\frac{x_{k+1}-x_{k}}{t_{k+1}-t_{k}}, \quad a_{k}=\frac{\Delta v_{k}}{\Delta t_{k}}=\frac{v_{k+1}-v_{k}}{t_{k+1}-t_{k}}
$$

At $t_{k}^{\prime}$ in the rocket, then, let

$$
v_{k}^{\prime}=\frac{\Delta x_{k}{ }^{\prime}}{\Delta t_{k}{ }^{\prime}}=\frac{x_{k+1}-x_{k}{ }^{\prime}}{t_{k+1}{ }^{\prime}-t_{k}{ }^{\prime}}, a_{k}^{\prime}=\frac{\Delta v_{k}{ }^{\prime}}{\Delta t_{k}{ }^{\prime}}=\frac{v_{k+1}{ }^{\prime}-v_{k}{ }^{\prime}}{t_{k+1}-t_{k}{ }^{\prime}}
$$

Direct substitution of (3.1) and (3.2) into (3.3) and (3.4) yields

$$
v_{k}^{\prime}=\frac{c^{2}\left(v_{k}-u\right)}{c^{2}-u v_{k}}, v_{k}=\frac{c^{2}\left(v_{k}^{\prime}+u\right)}{c^{2}+u v_{k}^{\prime}}
$$

$$
\mathrm{a}_{k}{ }^{\prime}=\frac{c^{3}\left(c^{2}-u^{2}\right)^{\frac{3}{2}}}{\left(c^{2}-u v_{k+1}\right)\left(c^{2}-u v_{k}\right)^{2}} \mathrm{a}_{k} \quad, \quad \mathrm{a}_{k}=\frac{c^{3}\left(c^{2}-u^{2}\right)^{\frac{3}{2}}}{\left(c^{2}+u v_{k+1}{ }^{\prime}\right)\left(c^{2}+u v_{k}\right)^{2}} \mathrm{a}_{k}{ }^{\prime}
$$

In the limit, (3.3)-(3.6) converge to their correct continuum counterparts. 
We turn finally to $(2.2),(2.3)$. At $t_{k}$ the lab, we approximate $(2.2)$ by the arithmetic formula

$$
F_{k}=\frac{m\left(t_{k}\right) c^{2}}{\left[\left(c^{2}-v_{k}^{2}\right)\left(c^{2}-v_{k+1}^{2}\right)\right]^{\frac{1}{2}}} a_{k}, m\left(t_{k}\right)=\frac{c m_{o}}{\sqrt{c^{2}-v_{k}^{2}}}
$$

We now have a basic theorem.

Theorem 1 If in the rocket

$$
m^{\prime}\left(t_{k}{ }^{\prime}\right)=\frac{c m_{o}}{\sqrt{c^{2}-\left(v_{k}\right)^{2}}}
$$

and if $F_{k}{ }^{\prime}=F_{k}$, then under the Lorentz transformation (3.7) is covariant, that is, it maps into

$$
F_{k}^{\prime}=\frac{m^{\prime}\left(t_{k}{ }^{\prime}\right) c^{2}}{\left[\left(c^{2}-\left(v_{k}{ }^{\prime}\right)^{2}\right)\left(c^{2}-\left(v_{k+1}\right)^{2}\right)\right]^{\frac{1}{2}}} a_{k}{ }^{\prime}
$$

Proof . The proof follows by direct substitution of (3.3) into the righthand-side of (3.7) and algebraic simplification using the mass formulas.

Once force $F$ is given, trajectory computation in the lab proceeds as follows. First normalize so that $m_{o}=c=1$. Then (3.3) and (3.7) are rewritten in the forms

$$
\begin{gathered}
x_{k+1}=x_{k}+\left(t_{k+1}-t_{k}\right) v_{k} \quad, k=0,1,2, \cdots \\
v_{k+1}=v_{k}+\left(t_{k+1}-t_{k}\right)\left(1-v_{k}^{2}\right)\left(1-v_{k+1}^{2}\right)^{\frac{1}{2}} F_{k}, k=0,1,2 \cdots .
\end{gathered}
$$

Unfortunately, (3.11) is implicit in $v_{k+1}$. This impediment is, however, easily removed by solving for $v_{k+1}$ explicitly. This is done by writing (3.11) as

$$
v_{k+1}-v_{k}=\left(t_{k+1}-t_{k}\right)\left(1-v_{k}^{2}\right)\left(1-v_{k+1}^{2}\right)^{\frac{1}{2}} F_{k}
$$

squaring both sides, and solving the resulting quadratic equation for $v_{k+1}$. of the two resulting roots, one is extraneous and the unique solution is 


$$
v_{k+1}=\frac{v_{k}+\left(t_{k+1}-t_{k}\right)\left(1-v_{k}^{2}\right) F_{k} \sqrt{1-v_{k}^{2}+\left(t_{k+1}-t_{k}\right)^{2}\left(1-v_{k}^{2}\right)^{2} F_{k}^{2}}}{1+\left(t_{k+1}-t_{k}\right)^{2}\left(1-v_{k}^{2}\right)^{2} F_{k}^{2}}
$$

In the limit, (3.7) and (3.9) converge to equations which are entirely equivalent to $(2.2)$ and $(2.3)$.

Thus, in the lab, motion of a particle $P$, from given initial data, is generated uniquely by $(3.10)$ and $(3.12), k=0,1,2, \cdots$. In the rocket, the would be calculated from

$$
x_{k+1}{ }^{\prime}=x_{k}{ }^{\prime}+\left(t_{k+1}{ }^{\prime}-t_{k}^{\prime}\right) v_{k}^{\prime}
$$

$$
v_{k+1}^{\prime}=\frac{v_{k}{ }^{\prime}+\left(t_{k+1}{ }^{\prime}-t_{k}{ }^{\prime}\right)\left(1-\left(v_{k}\right)^{2}\right) F_{k}{ }^{\prime} \sqrt{1-\left(v_{k}\right)^{2}+\left(t_{k+1}{ }^{\prime}-t_{k}{ }^{\prime}\right)^{2}\left(1-\left(v_{k}\right)^{2}\right)^{2}\left(F_{k}\right)^{2}}}{1+\left(t_{k+1}{ }^{\prime}-t_{k}\right)^{2}\left(1-\left(v_{k}\right)^{2}\right)^{2}\left(F_{k}\right)^{2}}
$$

We now come to our major theorem.

Theorem 2 Computations in the lab and the rocket frames of the motion of a particle $P$ of mass $m$ acted upon by a given force are related by the Lorentz transformation.

Proof Assume that the initial data $x_{o}, v_{o}, x_{o}^{\prime}, v_{o}^{\prime}$ are related by the Lorentz transformation. Hence

$$
\begin{array}{r}
x_{o}^{\prime}=\frac{x_{o}-u t_{o}}{\left(1-u^{2}\right)^{\frac{1}{2}}} \\
v_{o^{\prime}}=\frac{v_{o}-u}{1-u v_{o}}
\end{array}
$$

Now, by $(3.13)$

$$
x_{1}{ }^{\prime}=x_{o}{ }^{\prime}+\left(t_{1}^{\prime}-t_{o}{ }^{\prime}\right) v_{o}^{\prime}
$$

Suppose that under the Lorentz transformation $x_{1}{ }^{\prime}$ maps into $\bar{x}_{1}$. Then

$$
x_{1}{ }^{\prime}=\frac{\bar{x}_{1}-u t_{1}}{\left(1-u^{2}\right)^{\frac{1}{2}}}
$$

We need only show that $\bar{x}_{1}=x_{1}$. 
Hence, $(3.15)-(3.18)$ and

$$
t_{o}^{\prime}=\frac{t_{o}-u x_{o}}{\left(1-u^{2}\right)^{\frac{1}{2}}}, t_{1}^{\prime}=\frac{t_{1}-u \bar{x}_{1}}{\left(1-u^{2}\right)^{\frac{1}{2}}}
$$

yield

$$
\begin{aligned}
\frac{\bar{x}_{1}-u t_{1}}{\left(1-u^{2}\right)^{\frac{1}{2}}} & =x_{0}{ }^{\prime}+v_{0}{ }^{\prime}\left(t_{1}{ }^{\prime}-t_{0}{ }^{\prime}\right) \\
& =\frac{x_{0}-u t_{0}}{\left(1-u^{2}\right)^{\frac{1}{2}}}+\left(\frac{v_{0}-u}{1-u v_{0}}\right)\left(t_{1}-t_{0}+u x_{0}-u \bar{x}_{1}\right) .
\end{aligned}
$$

Hence

$$
\bar{x}_{1}-u t_{1}=x_{0}-u t_{0}+\left(\frac{v_{0}-u}{1-u v_{0}}\right)\left(t_{1}-t_{0}+u v_{0}-u \bar{x}_{1}\right)
$$

or

$$
\left(\bar{x}-u t_{1}\right)\left(1-u v_{o}\right)=\left(x_{0}-u t_{0}\right)\left(1-u v_{0}\right)+\left(v_{0}-u\right)\left(t_{1}-t_{0}+u x_{0}-u \bar{x}_{1}\right),
$$

which simplifies to

$$
\left(\bar{x}_{1}-x_{0}\right)\left(1-u^{2}\right)=v_{0}\left(1-u^{2}\right)\left(t_{1}-t_{0}\right)
$$

Thus,

$$
\bar{x}_{1}=x_{0}+\left(t_{1}-t_{0}\right) v_{0}
$$

so that, by (3.10), $\bar{x}_{1}=x_{1}$ and, by induction it follows that $x_{k+1}{ }^{\prime}$ and $x_{k+1}$ are related by the Lorentz transformation.

Let us show next that $v_{k+1}$ and $v_{k+1}$ are related by the Lorentz transformation. For $k=0,(3.14)$ implies

$$
v_{1}^{\prime}=\frac{v_{0}{ }^{\prime}+\left(t_{1}{ }^{\prime}-t_{0}{ }^{\prime}\right)\left(1-\left(v_{0}{ }^{\prime}\right)^{2}\right) F_{0}{ }^{\prime} \sqrt{1-\left(v_{0}{ }^{\prime}\right)^{2}+\left(t_{1}{ }^{\prime}-t_{0}{ }^{\prime}\right)^{2}\left(1-\left(v_{0}\right)^{2}\right)^{2}\left(F_{0}{ }^{\prime}\right)^{2}}}{1+\left(t_{1}{ }^{\prime}-t_{0}\right)^{2}\left(1-\left(v_{0}{ }^{\prime}\right)^{2}\right)^{2}\left(F_{0}{ }^{\prime}\right)^{2}}
$$

Substitution of 


$$
t_{1}^{\prime}=\frac{t_{1}-u x_{1}}{\left(1-u^{2}\right)^{\frac{1}{2}}}, t_{0}^{\prime}=\frac{t_{0}-u x_{0}}{\left(1-u^{2}\right)^{\frac{1}{2}}}, x_{0}{ }^{\prime}=\frac{x_{0}-u t_{0}}{\left(1-u^{2}\right)^{\frac{1}{2}}}, v_{0}{ }^{\prime}=\frac{v_{0}-u}{1-u v_{0}}
$$

and

$$
F_{0}^{\prime}=F_{0}=\frac{v_{1}-v_{0}}{\left(t_{1}-t_{0}\right)\left(1-v_{0}^{2}\right)\left(1-v_{1}^{2}\right)^{\frac{1}{2}}}
$$

into (3.19) yields, after simplification,

$$
v_{1}^{\prime}=\frac{\left(v_{1}-u\right)\left[\left(1-v_{0} v_{1}\right)\left(1-u v_{0}\right)+\left(v_{1}-v_{0}\right)\left(u-v_{0}\right)\right]}{\left(1-u v_{1}\right)\left[\left(1-v_{0} v_{1}\right)\left(1-u v_{0}\right)+\left(v_{1}-v_{0}\right)\left(u-v_{0}\right)\right]}
$$

The theorem will follow then from (3.20), since the terms in the brackets are identical, provided these terms are not zero. However, this is the case and we will show it next, but recall that all velocities under consideration are less than the speed of light. Hence, since $|u|<1$ and $|v|<1$, then

$$
\begin{gathered}
\left(1-u^{2}\right)\left(1-v^{2}\right)>0 \\
\left(u^{2}-1\right)\left(1-v^{2}\right)<0 \\
u^{2}+v^{2}-1-u^{2} v^{2}<0 \\
u^{2}+v^{2}<1+u^{2} v^{2} \\
u^{2}-2 u v+v^{2}<1+u^{2}-2 u v \\
(u-v)^{2}<(1-u v)^{2}
\end{gathered}
$$

so that

$$
|u-v| \leq|1-u v|=1-u v
$$

Hence

so that

$$
\left|v_{1}-v_{0}\right|<1-v_{0} v_{1} \quad,\left|u-v_{0}\right|<1-u v_{o}
$$

$$
\left(1-v_{0} v_{1}\right)\left(1-u v_{0}\right)>\left|v_{1}-v_{0}\right| \cdot\left|u-u_{0}\right| \geq-\left(v_{1}-v_{0}\right)\left(u-u_{0}\right) .
$$

Thus

$$
\left(1-v_{0} v_{1}\right)\left(1-u v_{0}\right)+\left(v_{1}-v_{0}\right)\left(u-v_{0}\right)>0,
$$

so that the terms in the brackets in (3.20) cancel. 
4. A Relativistic Harmonic Oscillator As in Newtonian mechanics, we now define a harmonic oscillator in the lab frame to be a particle $P$ of mass $m$ upon which acts a force $F=-K^{2} x$. For simplicity $K$ is normalized to unity. Assume then that $x(0)=x_{0}=0$ and $v(0)=v_{0}$. With $\Delta t=0.0001$, the motion of $P$ was generated using (3.10) and (3.11) for 30,000 times for each of the cases $v_{0}=0.001,0.01,0.05,0.1,0.3,0.5,0.7,0.9$.

Fig. 1 shows the amplitude and period of the first complete oscillation for the case $v_{0}=0.001$. For such a relatively low velocity, the oscillator should behave like a Newtonian oscillator and, indeed, this is the case, with the amplitude being 0.001 and, to two decimal places, the period being $6.28(\sim 2 \pi)$. Subsequent motion of this oscillator continues to show almost no change in amplitude or period. At the other extreme, Fig. 2 shows the motion for $v_{0}=0.9$, which is relatively close to the speed of light. To two decimal places, the amplitude of the first oscillation is 1.61 while the period is 8.88. These results are distinctly non-Newtonian, and, to thirty thousand time steps, these results remain constant to two decimal places but do show small increments in the third decimal place. Finally, in Fig. 3 is shown how the amplitude of a relativistic oscillator deviates from that of a Newtonian oscillator with increasing $v_{0}$. 


\section{References}

1. D. Greenspan, Arithmetic Applied Mathematics, Pergamon, 0xford, 1980 .

2. J. Abrycht and A. Marciniak, "Discrete Dynamical Equations in Minkowski Space", Int. J. Theor. Phys., 20, 1981, p.821.

3. H.S. Snyder, "Quantized Space Time", Phys. Rev., 71, 1947, p. 38.

4. T.D. Lee, "Can Time Be a Discrete Dynamical Variable?", Phys. Letters, 122B, 1983, p.217.

5. J.C. Jackson, "A Quantization of Time", J. Phys. A, 10, 1977, p. 2115.

6. A. Horzela, E. Kapuscik, J. Kempczynski, and C. Uzes, "On Discrete Models of Space-time", Prog. Theor. Phys., 88, 1992, p. 1065.

7. H. Yamamoto, "Quantum Field Theory on Discrete Space-time", Phys. Rev., D30, 1984, p. 1727 .

8. H. Arzelies, Relativistic Kinematics, Pergman, 0xford, 1966

9. P. Bergmann, Introduction to the Theory of Relativity, Prentice-Hall, Englewood Cliffs, N.J., 1942.

10. R. Resnick, Basic Concepts of Relativity and Early Quantum Mechanics, Wiley, N.Y., 1972 .

11. H.M. Schwartz, Introduction to Special Relativity, McGraw-Hill, N.Y., 1968.

12. J.L. Synge, Relativity: The Special Theory, North-Holland, Amsterdam, 1965 . 